\title{
MODERN APPROACHES TO MEDICAL AND PSYCHO- LOGICAL SUPPORT OF FAMILIES WITH INFANTS WITH SEVERE CRANIOCEREBRAL TRAUMA
}

\author{
Kharkiv National Medical University Kharkiv, Ukraine
}

\begin{abstract}
The study involved a comprehensive survey of 137 families (mother and father) of children with severe somatic disease aimed at the development and evaluation of the system of medical and psychological support of families with a somatically challenged child. The main group comprised 97 families participating in the program of medical and psychological support, and the control group included 40 families who did not receive psychological support. According to our findings, a serious disease of the child became a psychotraumatic situation for all the parents, resulting in the development of anxiety-depressive reactions and conditions. Psychodiagnostic examination showed that the parents had mild, moderate depressive and anxious episodes on the Hamilton Rating Scale; high levels of situational and personal anxiety according to the C.D. Spielberger Inventory, a high level of neuropsychic stress on T.A. Nemchin Scale. The couples under investigation noted tensions in family relationships, family conflicts, related to the treatment of the child and escalation of pre-existing interpersonal and marital problems that led to distancing and a decrease in internal family resource. Based on these data, we have developed a system of medical and psychological support of the families with somatically challenged child, which consisted of four consecutive phases and included the use of individual cognitive-behavioral therapy (Beck AT, 2006), family therapy (Eidemiller E. G., 2003), rational therapy (classic Dubois P., 1912) and psychological educational programs. Case monitoring in the main group following the employment of the proposed system of medical and psychological support showed a stable positive pattern of psychological state with a statistically significant total reduction of anxiety-depressive states and the harmonization of the marital relationship.
\end{abstract}

KeyWords: Medical and psychological support, anxiety, depression, family interactions, infants with severe craniocerebral trauma.

\section{INTRODUCTION}

Protection of mental and physical health of the mother and child is one of the priorities of the state social policy in our country. Epidemiological studies of craniocerebral trauma incidence in children are conducted in many countries and indicate general trends with slight fluctuations in rates. In the CIS countries this figure is about $2 \%$. Traumatic brain damage in newborns is 2\%, 25 - 25.9\% in infants, 7.1 $8 \%$ in toddlers, $20 \%$ in preschool age children and $45 \%$ in school age children. Of all the children affected by craniocerebral trauma infants comprise $27.9 \%$ [1, 2, 3]. Serious diseases in children have a psycho-traumatic effect on parents, manifested by disorders of adaptation and anxiety-depressive states.

\section{Corresponding Author:}

Oleksandr Dukhovskyy, MD, Head of Children's Neurosurgery Department of Kharkiv City Clinical Hospital of Urgent and Emergency Medical Aid, Ukraine.
Craniocerebral trauma is one of the most important and actual problems of pediatric traumatology, which has great social and medical significance due to its prevalence and severity of consequences [4, 5]. Parents of an infant with severe craniocerebral trauma are under high psychological pressure, resulting in psycho-emotional impairments and other manifestations of psychosocial maladaptation. During this period, it is particularly relevant to search for sources of medical and psychological support, to increase the psychological adaptation resource of parents [6, 7].

Psycho-emotional state of the parents is closely associated with changes in physical and mental condition of a sick child. At the same time, psychological well-being of the child depends on the mental state of his parents, in particular, those who are emotionally closer. Thus, a serious illness of a child is a powerful stressful situation for the whole family, which dramatically changes family functioning $[8,9,10]$.

Destabilization occurs even in resourceful and welladapted family-based systems; however, family has a pow- 


\section{RESULTS AND DISCUSSION}

The findings showed that craniocerebral trauma in infant was a traumatic situation for all the parents, which provoked a whole range of difficult issues, namely the need for decisions related to the treatment of the child; concern about the impact of injury and surgical treatment on physical and mental development of the child; experiences associated with planned operational intervention; disruption of family functioning; aggravation of family, marital, interpersonal and intrapersonal conflicts; the need for adaptation to stay in a medical institution (medical procedures, everyday aspect); exclusion from the professional activities of the caring family member.

The most significant issues for parents at the stage of admission to neurosurgical hospital and examination of the child were the questions of actual surgical intervention, effectiveness of treatment and its impact on physical and mental development of the child; concern over the result of the operation, its consequences; fear of anesthesia, its side effects.

In the postoperative period, the most severe concern is the care, rehabilitation and exclusion from social functioning in need of the child's care, redistribution of responsibilities at the time of treatment. Parents had to adapt to conditions of functioning in the hospital and adapt the child to them.

The range of stress experiences varied in women and men. While staying at a hospital, the mother was near the sick child and her duties included care for the child, while men were forced to engage in financial and social aspects.

According to the findings obtained in the study, parents (mother and father) had low mood (97.6\% of patients), depression (66.2\%), a sense of sadness and sorrow $(73.5 \%$ of respondents), anxiety, constant internal stress with inability to relax $(67.4 \%)$, state of confusion (36.3\%), fears and concerns associated with weather conditions (87.3\%), a sense of danger and failure (69.8\%).

According to the Hamilton Depression Rating Scale, 53.6\% of the surveyed had mild (16.4 \pm 1.3 points) and $32.3 \%$ had moderate ( $24.6 \pm 1.7$ points) depressive episodes. According to the Hamilton Anxiety Rating Scale, $55.4 \%$ of the parents

There is no conflict of interests. 
had mild (16.6 \pm 1.3 points) and $40.2 \%$ had moderate (24.9 \pm 1.8 points) anxiety episodes.

Psychodiagnostic assessment showed high levels of situational and personal anxiety in the surveyed parents by $C$. $D$. Spielberger method (58.89 and 59.19 points, respectively) with higher rates among mothers, high level of severity of mental stress on the scale of T.A. Nemchin (69.41 points).

Analysis of applied coping strategies concerning behavioral method helping to cope with the situation, showed that most families used strategies aimed at emotion ( $57.1 \pm 5.6$ points), avoidance (51.7 \pm 5.7 points), social distraction and adjustment ( $50.9 \pm 7.2$ points, respectively), $\mathrm{p}<0.05$.

The families under investigation noted tension in family relationships, family conflicts, both related to the treatment of the child, and escalation of previously existing interpersonal and marital problems, which led to distancing and reducing the intra-family resource.

Thus, consequences of an infant's severe craniocerebral trauma for parents included development of intense psychological reactions to the stressful situation (82.3\%); transformation of psychological reactions into expressed anxietydepressive disorder (79.4\%); maladaptive behavior during the stages of treatment (61.1\%); psychosocial maladjustment (40.1\%); disturbance of interpersonal relationships (87.2\%); deformation of family interaction (72.3\%).

According to our findings, we have elaborated a system of medical and psychological support for families of infants with severe craniocerebral trauma, which was regulated by the targets of medical psychological effects and consisted of four consecutive phases: 1st phase - compliance development, aimed at the establishment of a productive contact between the physician and the parents of the infant with severe craniocerebral trauma; 2nd phase - correction of intense mental reactions to stressful situations and change in anxious and depressive attitudes, formation of adequate ideas on postoperative period prognosis, effectiveness of treatment and impact on physical and mental development of the child; 3rd phase - the stage of correction of emotional responses to the child's condition, surgery, the need to stay in hospital, aimed at reduction of anxiety and depressive symptoms, correction of family relations; the 4th phase - establishment and maintenance of the results by potentiation of positive emotions, fixation on the improved health of the child, specifics of rehabilitation.

The psychotherapeutic complex of the proposed system of medical and psychological support included the use of individual cognitive-behavioral therapy (A. T. Beck, 2006), family therapy (E. G.Eidmiller, 2003), rational psychotherapy (classic version by P. Dubois, 1912).

Psychological education was chosen to be the sense-making element of the developed model. Psycho-educational classes were held in closed groups of 8 to 10 parents, lasting for 45 minutes (twice a week during the entire stay of the parents with the child in the neurosurgical department). The main issues proposed during psychological education included the concept of craniocerebral trauma, the necessity and methods of neurosurgical treatment, the needs of the child during treatment, possible consequences (physical and mental) of craniocerebral trauma, psychological state of the family members of the child; self-regulation of mental state.

Case monitoring in the main group following the employment of the proposed system of medical and psychological support showed a stable positive pattern of psychological state with a statistically significant total reduction of emotional disorders in parents, $\mathrm{p}<0.05$ : reduction in anxiety level in $73.5 \%$ of the mothers and in $83.6 \%$ of the fathers; leveling of depressive symptoms in $71.1 \%$ of the mothers and $88.6 \%$ of the fathers; a decrease in family conflicts in $72.5 \%$ of families; harmonization of marital relationship in $65.28 \%$ of the couples.

In the control group aggravation of psychopathological symptoms was diagnosed in $35.4 \%$ of the mothers and $11.2 \%$ of the fathers, $64.6 \%$ of the mothers and $88.82 \%$ of the fathers had stable presentation of anxiety-depressive disorders, and deterioration of family interactions was observed in $42.3 \%$ of the families.

\section{CONCLUSIONS}

The obtained results allowed us to substantiate the expedience of medical and psychological support of the families of infants with severe craniocerebral trauma. 


\section{REFERENCES}

1. Back A. L., Arnold R. M., Baile W. F. et al.(2007). Efficacy of communication skills training for giving bad news and discussing transitions to palliative care. Arch Intern Med., 167, 5, 453 - 460.

2. Salehpoor F., Bazzazi A. M., Estakhri R. et al. (2010). Correlation between catecholamine levels and outcome in patients with severe head trauma. Pak. J. Biol. Sci., 13, 15, 738-742.

3. Kobori N. Hu B., Dash P. K. (2011). Altered adrenergic receptor signaling following traumatic brain injury contributes to working memory dysfunction. Neuroscience, 172, 293-302.

4. Pearlin, L. I. (2012). The social contexts of stress. Handbook of stress. Theoretical and clinical aspects. Eds. L. Goldberger, S. Breznitz. N. Y.: The Free Press, 367-379.

5. Hung-Chen Wang, Tzu-Ming Yang, Yu-Jun Lin et al. (2014). Serial Serum Leukocyte Apoptosis Levels as Predictors of Outcome in Acute Traumatic Brain Injury. BioMed Research International, 11, Article ID 720870,

6. Polinder S., Haagsma J., Klaveren D. van. (2015). Health-related quality of life after TBI: a systematic review of study design, instruments, measurement properties, and outcome [Electronic Resource]. Population Health Metrics., 13(4), DOI: 10.1186 / s12963-015-0037-1.

7. Steinbuechel N. von, Covic A., Polinder S. (2016). Assessment of Health-Related Quality of Life after TBI: Comparison of a Disease-Specific (QOLIBRI) with a Generic (SF-36) Instrument [Electronic Resource]. Behavioral Neurol, 2016, DOI: 10.1155 / 2016/7928014

8. Valiullina S.A., Promyslova S.A. (2014).Children craniocerebral trauma: epidemiology and basic principles of the organization of medical care. Moscow, 24.

9. Kvasnitskiy M.V. (2013). Diagnostics and prescription of the first medical aid in cases of craniocerebral trauma. Medicine of Urgent Conditions, 2013, 3 (50), 34-38.

10. Kazakov V. Ye. (2014).Immune disorders as harbingers of the unfavorable course of craniocerebral trauma. Ukrainian Journal of Psychoneurology, 22, 1(78),
129-131.

11. Lin Hung-Chen Wang, Tzu-Ming Yang, Yu-Jun. (2014). Serial Serum Leukocyte Apoptosis Levels as Predictors of Outcome in Acute Traumatic Brain Injury. BioMed Research International, 2014, 720870, 11-13

12. Markova M.V., Piontkovskaya O. V. (2013). Functionality of the family system as a factor of the prevention of mental health disorders in parents of children with cancer pathology. International Neurological Journal, 4, 58, 49-53.

13. Piontkovskaya O. V. (2013). Behavioral Patterns to Overcome Stress in Parents of Oncologic Children. Journal of Psychiatry and Medical Psychology, 1, 32, 80 - 83.

Received: $\quad 08$ - Jun. - 2017

Accepted: $\quad 23$ - Sep. - 2017 